\title{
Maternal Healthcare Service Transformation: Exploring Opportunities for IT Use in Task Shifting
}

\author{
Hawa Nyende \\ University of Gothenburg, Sweden \\ hawa.nyende@ait.gu.se
}

\begin{abstract}
The transformation of healthcare services is expected to reduce health inequalities and to accelerate gains in health outcomes. Task shifting is one of the strategies adopted in healthcare transformation to make efficient use of human resources. However, limited research exists on how tasks are shifted beyond midwives, to involve community health workers or village health team members (VHTs) and pregnant women, and how IT supports and or triggers execution of shifted tasks. We examine the shifting of tasks in maternal healthcare, by interviewing midwives and VHTs in three districts in Uganda. Findings show four categories of tasks shifted at various levels of healthcare but with limited use of IT to execute tasks. We propose a model depicting opportunities for IT use both as an enabler and a trigger in executing tasks shifted. We recommend further investigations to identify IT opportunities that would trigger service exchange for pregnant women beyond health workers to include families and friends.
\end{abstract}

\section{Introduction}

Improving maternal healthcare is high priority for the world health organization (WHO) [1]. The unacceptably high maternal mortality rates can be reduced through strengthening healthcare systems and addressing inequalities in access to and quality of healthcare services [1]. Health systems strengthening calls for adopting the healthcare services delivery lens, which demands for new or renewed mechanisms, competences, and schemas for strategizing processes for service transformation [2].

Service transformation has been viewed from different perspectives including servitization, service dominant logic, resource-based view and dynamic capabilities [3]. Regardless of the perspective adopted, researchers agree that service transformation requires changes to the organizational structure, culture, competences and ways to delivering value to customers and stakeholders [3]. In healthcare, service transformation has been adopted through new healthcare models that are patient-centered [2]. Evidence has shown that involvement of patients in their treatment creates value as they actively seek and share information with health professionals, friends, family, support groups and colleagues to redesign their treatment programs [4] and prevent diseases through proper diet and exercises [5].

Task shifting has been adopted as an innovation to achieve effective healthcare coverage [6] through making more efficient use of human resources [7]. Task shifting has been implemented in many countries to improve maternal healthcare, by training lower level cadres to provide pregnancy and childbirth care [8]. In low resource settings, community health workers (CHWs) who are non-professionals are engaged in the strategy [7]. This is mainly because they bridge the formal health systems to communities by supporting the relevance, acceptability and accessibility of health services [7] to groups of people, such as pregnant women, who are difficult to reach [9].

The current model in which task shifting strategy operates is not fully patient-centered, but based on a 'traditional healthcare system in which health workers and CHWs provide services to patients which, leads to low quality of life' [4]. A systematic review by Dawson [10] shows that task shifting in maternal healthcare is focused on shifting clinical tasks among health workers instead of having shared health service delivery at all levels of care. This has caused the strategy to be opposed by patients and their families due to fear of predicted wrong medication and therefore requires acceptance from patients and their families [11]. In addition, the strategy faces challenges in continuous motivation of "shifted-to" cadres in form of incentives and appreciation, which may be difficult to achieve in the long run [11], [12]. Further still, when tasks are shifted to CHWs, they face barriers such as lack of appropriate tools to provide services and to collect data, limited access to training and supervision [9]. Without proper designs, task shifting may increase 
system costs by worsening overall population health due to poor clinical quality or increase the number of staff in healthcare system without changing careseeking patterns among patients [13].

To achieve a healthcare transformation with a task shifting strategy, there is need to understand how patients can be engaged in healthcare. Hardyman [14] recommends understanding patients engagement in healthcare through value co-creation. Co-creation of value in healthcare requires participation of various people in care delivery process [15] including the patient [16]. Value co-creation in healthcare occurs directly through interactions between patients and medical staff and indirectly through using technology systems for interactive exchange of information [15]. Information technology improves the quality of healthcare and transforms the way healthcare is delivered [17]. IT plays a dual role by triggering (operant) and enabling or facilitating (operand) actors to share resources [16]. The use of IT across the healthcare system and the engagement of patients through digital channels changes the way care is coordinated and provided [18]. However, there is a lack of studies that focus on the operant role of IT in organizations [19] and the role of technology in value co-creation [20].

Therefore, as part of healthcare transformation, the strategy needs to be rethought about in terms of patient-centeredness, by identifying tasks that can be shifted to patients and ways in which IT can trigger active participation and value co-creation in such a shift. Limited studies exist on shifting key clinical tasks among doctors, non-physician clinicians, nurses and midwives in maternal healthcare and few of them mention the involvement of CHWs in task shifting [10]. In addition, no studies have explicitly examined tasks that are shifted to patients in maternal healthcare and how IT can support execution of such tasks. This study seeks to examine the task shifting strategy in maternal healthcare to answer the research questions "Which tasks are shifted in maternal healthcare service delivery by whom and to who? What opportunities exist for IT to enable or trigger execution of shifted tasks?"

The study contributes by developing a model that depicts opportunities for IT use after tasks are shifted in maternal healthcare. This study addresses a call for research in task shifting in maternal healthcare specifically on ways to engage teams of workers and community members in delivering and advocating for interventions at various levels [10].

In the following sections, literature on opportunities for IT in healthcare transformation and task shifting strategy in maternal healthcare is provided. This is followed by the method, results, discussion and a proposed model for IT opportunities in task shifting.

\section{Literature review}

\subsection{Opportunities for IT in healthcare transformation}

Healthcare transformation requires adapting new healthcare models that are patient-centered [2]. Patient engagement in healthcare can be understood through value co-creation [14]. Value co-creation is defined as the processes or activities that underlie resource integration and incorporate different actor roles in the service ecosystem [16]. Research on involvement of patients in healthcare through value co-creation has received much attention [4], [15], [21], [22], mainly because it is viewed as an innovation that improves quality of life [4]. Value co-creation can happen within and outside hospital activities to include family and friends and through self-generated activities by patients [4], [22]. Value co-creation is supported through the dual role of IT, as an operant and operand [16].

IT transforms healthcare by shifting data collection from clinicians to patients, making actionable use of data collected and adopting new operating and business models [17]. In healthcare, the dual role of IT improves service provision, diagnosis and treatment, healthcare coverage and decision making [23]. Patient-centered exchange models enable sharing of patient data and laboratory results with patients as required [24]. Health online communities play an operant role of triggering rural patients to communicate and share knowledge with their peers, to maintain a positive outlook and better manage their disease conditions [25]. In addition, e-health kiosks improve healthcare practices among illiterate villagers [26]. IT has been used to build social networks that promote wellness behaviors, preventive care and timely seeking of corrective healthcare [26]. The role of IT as an operand enables access to information and acquisition of knowledge for patients to participate in the co-creation [27] and enables health workers to exchange patient data for care continuity [28].

In low resource settings with limited infrastructure, CHWs use mHealth tools as enablers in data collection and reporting, decision support, training and supervision [29] and emergency referrals through improved communication between pregnant women and health workers [30]. They also use mHealth tools as triggers to alert and remind patients [29]. In addition digitalized prediction models trigger service exchange by identifying complications [31]. 


\subsection{Task shifting strategy in maternal healthcare}

Healthcare systems can be strengthened by improving numbers and skills of the health workforce [7]. In order to strengthen and extend the workforce to rural areas, the World Health Organization (WHO) in collaboration with the United States Global AIDS Coordinator (OGAC) launched a task shifting strategy [7]. The main contribution of the strategy was to make more efficient use of human resources [7]. The strategy enables healthcare professionals such as doctors and specialized clinicians to move tasks to less trained and qualified health practitioners, such as nurses and CHWs [32] or village health team members (VHTs). Task shifting aims at enhancing quality and reducing costs [11], which can be achieved through increased service provision, equivalent health professional performance across cadres and patient outcomes [10].

Perspectives on task shifting is where 1.) tasks shifted are defined, described and funds are moved to the individual assigned the task and, 2.) task shifting is viewed as delegation of specific tasks or substituting a health worker type for another [10]. This study adopts the later view of task shifting where tasks are delegated to lower cadres to free up senior midwives and midwives so that they can provide more complex care.

In maternal healthcare, tasks can be shifted from maternity doctors to qualified nurses, other medical doctors and CHWs [11]. Specifically, tasks can be shifted from midwives to other nurses (horizontal shift) or to nursing assistant and CHWs (vertical shift), and from doctors to senior midwives (vertical shift) [33]. In addition, task shifting also involves attendants and patients' relatives in roles such as feeding and bathing inpatients, taking specimens and collection of results from laboratories [34]. However, most studies focus on shifting of tasks to midwives rather than from midwives [33].

The WHO developed guidelines on optimizing healthcare roles to improve access to key maternal and newborn interventions through task shifting [35], from which we categorize three types of tasks. Namely, tasks that do not require cadres to have formal professional training and certification in maternal healthcare but have limited training on the task to be carried out by them (here in known as non-technical), for example promotional tasks. Tasks that require cadres to have some training in maternal healthcare but can be taken on by such cadres with rigorous research and monitoring and evaluation (here in known as intermediate), for example, administration and distribution of oral supplements to pregnant women. Tasks that require cadres to have formal professional training and certification in maternal healthcare to be carried out by them (here in known as technical), for example delivery of injectable antibiotics for preterm membrane rupture. In addition, there are complex (herein known as clinical) tasks that are less related to pregnancy or delivery and are shifted to midwives, examples include genetic and cervical cancer screening, abortion services and other sexual and reproductive services [33]. WHO recommends nontechnical and some intermediate tasks to be carried out by CHWs and technical tasks to be carried out by midwives in the task shifting strategy [35].

Task shifting in maternal healthcare is challenged with poor staff coordination and preparation, low skills, provider absence and resistance, lack of equipment and drugs [10], poor integration of new tasks into the broader organization of care [33], inadequate guidelines, heavy workloads and high disease burden [34]. Midwives express other concerns of uncertain liability and fear of missing rare abnormalities [33].

Expanding access to maternal healthcare services through the task shifting strategy requires an interplay of different components including policy and regulatory support, determination of roles and responsibilities, determination of qualifications, education and training and service delivery support [8] [9]. However, there is need to redesign the strategy to achieve healthcare transformation.

\section{Method}

In this section, the method used to conduct the study is described. The research approach, study setting, selection of participants, data collection and analysis procedures are described below.

\subsection{Research approach and study setting}

This paper reports for a study based on a case study approach [36] to investigate how tasks are shifted in maternal healthcare and opportunities for IT after tasks are shifted. Case study approach was used because it enables researchers to develop concepts, generate theory, draw specific implications and gain rich insights [36] on the task shifting strategy and in order to explore opportunities for IT use in maternal healthcare.

The study was conducted in three districts in Uganda. Uganda was selected because it is one of the developing countries with a high maternal mortality rate of 343 per 100,000 live births [37] and has taken on task shifting for decades [34]. Districts were selected based on location so as to understand if the infrastructure in the different settings could affect the 
use of technologies to support tasks that were being shifted. One of the districts (District A) was selected because the district is located in the rural area that is hilly with poor road infrastructure. Pregnant women have to walk long distances to health facilities for care and the district has registered high rates of teenage pregnancies. Due to its rural setting, we find more use of VHTs who provide services to pregnant women in communities. District B is located near the capital city with two health facilities near the district offices and good network and road infrastructure. District $\mathrm{C}$ was selected because the hospital in this district serves as the regional hospital for district $\mathrm{B}$ and district $\mathrm{C}$, and a lot of collaborations take place through referrals. Another reason why districts were selected is because it was easy to get access to health workers in these districts through the assistant district health officers (ADHO). ADHOs are in charge of all maternal and child health care activities in the respective districts.

\subsection{Data collection and selection of participants}

Qualitative data collection was used in which, interviews were held with health workers who are shown in Table 1. Permission was obtained from ADHOs to visit health facilities in order to interview participants. Administrators in health facilities communicated with participants well in advance before the interviews took place. A total of 23 interviews were conducted. Questions in the interview guide focused on getting insights on how the task shifting strategy works, its negative and positive effects on maternal healthcare service delivery, how patients are involved in the task shifting strategy and opportunities in which IT supports health workers after tasks are shifted to them. Specifically, questions included qualifications, roles and responsibilities of health workers, how roles are shifted among health workers and if there are roles shifted to patients, resources used to provide maternal healthcare services, IT support systems, training and supervision, motivation and reward systems and, referral systems in maternal healthcare.

Health workers were selected based on the districts in which they work and their professional roles in maternal healthcare. This was done to understand how tasks are shifted between healthcare providers including senior midwives or nursing officers, midwives and VHTs. Midwives provide healthcare to pregnant women and closely interact with village health team members (VHTs) and gynecologists or obstetricians during service provision. VHTs work as intermediaries between midwives and pregnant women and provide services to pregnant women in communities. Senior midwives and midwives sometimes take on the role of in-charge at the health facilities and supervise midwives. Records officers collaborate with midwives in managing records and reporting on maternal healthcare services.

\subsection{Data analysis method}

To analyze the qualitative data, thematic analysis method was used to "identify, analyze and report patterns within the data" [38]. A step-by-step guide by Braun and Clark [38] was followed during the analysis. The steps were familiarizing with the data, generating initial codes from the data, searching for themes, reviewing themes, defining and naming themes and producing the report. As a first step, interviews were recorded and transcribed.

\section{Table 1. Study participants interviewed}

\begin{tabular}{|l|l|c|}
\hline & Participants & Number \\
\hline \multirow{3}{*}{$\begin{array}{l}\text { District } \\
\text { A }\end{array}$} & $\begin{array}{l}\text { Senior Nursing Officer (SNO) } \\
\text { / senior midwife }\end{array}$ & 3 \\
\cline { 2 - 3 } & Midwives & 5 \\
\cline { 2 - 3 } & Village Health Team (VHTs) & 6 \\
\hline \multirow{2}{*}{\begin{tabular}{l} 
Bistrict \\
\cline { 2 - 3 }
\end{tabular}} & Senior Nursing Officer (SNO) & 1 \\
\cline { 2 - 3 } District & Midwife & 1 \\
\cline { 2 - 3 } & Records officer & 3 \\
\cline { 2 - 3 } & $\begin{array}{l}\text { In-charge Maternity / Senior } \\
\text { midwife }\end{array}$ & 1 \\
\cline { 2 - 3 } & Midwives & 2 \\
\cline { 2 - 3 } & Records officer & 23 \\
\hline & & \\
\hline
\end{tabular}

Using Nvivo, transcriptions were imported and used to identify, name and categorize phrases and words in order to develop initial codes. Initial codes were reviewed and refined to form final codes that were grouped into themes. Themes were reviewed iteratively with reference to initial codes. Themes were refined and named to generate final themes. Final themes included categories of tasks shifted, in which we placed non-technical, intermediate, technical and clinical tasks and, IT support, in which we placed dual roles of IT (as an enabler and a trigger).

\section{Results}

Results present insights on the tasks shifted among midwives, VHTs and pregnant women, and how IT is used as a trigger and an enabler to execute tasks.

\subsection{Categories of tasks shifted}


Task shifting is viewed as delegation of tasks in this context and it occurs without a formal plan in place. Tasks shifted are categorized into non-technical, intermediate, technical and clinical tasks. Senior midwives shift tasks to lower level cadres such as midwives. In addition, midwives shift tasks to VHTs and, midwives and VHTs shift tasks to pregnant women as shown in Table 2 and elaborated below:

\section{Non-technical tasks}

Non-technical tasks are carried by midwives, VHTs and pregnant women but mostly by VHTs as shown in Table 2. Such tasks mainly include administrative, data collection, promotional tasks, distribution and monitoring.

Non-technical tasks shifted from senior midwives to midwives are mainly administrative and include ordering drugs, assigning tasks to fellow midwives and scheduling. Non-technical tasks mainly data capture, promotional activities, distribution and monitoring have been shifted from midwives to VHTs as shown in Table 2. Midwives delegate promotional tasks to VHTs because they feel that pregnant women will listen to VHTs more than themselves. As quoted from midwife district A “...some pregnant women think we are telling them to bring birth materials for our use yet it is for them ... I think if the VHTs are the ones to tell them, they can know that it is for their use only"

Other tasks mainly promotional have been shifted by midwives and VHTs to pregnant women as shown in Table 2. Midwives delegate tasks to women leaders (known as mentor mothers) to provide health talks to fellow women at health facilities as quoted by one of the midwives from district $\mathrm{B}$ "...we divide mothers into family support groups, and we have mentor mothers in each group who teach others about any topic such as nutrition". The reasons why midwives use mentor mothers is to avoid stigma among new mothers especially those who are pregnant and are infected with HIV/AIDs as a way of prevention of mother to child transmission (PMTCT).

In addition, midwives and VHTs tell pregnant women to prepare for delivery through savings, exercising and dieting as quoted from a VHT, "...women should engage in exercises such as digging and should remain healthy, and earn money to support them in delivery." Birth preparedness is also emphasized through community laws, community dialogues and worshipping centers. VHTs recommend trainings to pregnant women through medical outreaches as another mechanism for women to save money in case of emergencies. VHTs expressed need for pregnant women to take on the responsibility of having regular checkups without waiting for VHT reminders and should take dieting as an important task. As quoted by a VHT "when a pregnant mother is malnourished you find them with swelling legs ... they should take responsibility to always feed well"

Intermediate tasks

Intermediate tasks mainly include supervision, testing and immunization. Supervision is the only intermediate tasks shifted to midwives. Midwives also shift the immunization task to VHTs, which they carry out under their supervision. As quoted by a VHT "Wednesdays when we have immunization, you find there's one nurse available to do the work as VHTs we come in to help like recording the information and helping the nurse to do the immunization".

\section{Technical tasks}

Technical tasks mainly diagnosis, review and treatment of pregnant women are roles of midwives including care for pregnant women, discharging mothers and reporting. However, technical tasks for complicated cases are shifted to midwives from senior midwives and doctors. These include handling complicated cases like breach deliveries and reviewing mothers.

\section{Clinical tasks}

Clinical tasks include testing and prescription. These tasks are supposed to be carried out by lab technicians and doctors respectively but they are shifted to midwives. A quote from midwife from district B "I test the mothers... that work is for the lab, but when they come because we don't want the mothers to overstay due to many clients, I test them." In addition, midwives also prescribe medicine as quoted from midwife district A "prescribing for the mothers, should either be a physician or a doctor but sometimes I prescribe."

Table 2. Shifting of tasks

\begin{tabular}{|c|c|}
\hline $\begin{array}{l}\text { Health } \\
\text { provid } \\
\text { er }\end{array}$ & Tasks shifted to them by higher levels \\
\hline $\begin{array}{l}\text { Midwi } \\
\text { ves }\end{array}$ & $\begin{array}{l}\text { Non-technical tasks } \\
-\quad \text { Ordering drugs } \\
-\quad \text { Assigning tasks to fellow midwives } \\
-\quad \text { Scheduling } \\
\text { Intermediate tasks } \\
-\quad \text { Supervision } \\
\text { Technical tasks (Roles and shifted tasks) } \\
-\quad \text { Care for pregnant women } \\
-\quad \text { Discharging mothers } \\
-\quad \text { Reporting } \\
-\quad \text { Complicated cases Reviewing mothers } \\
\text { Clinical tasks } \\
-\quad \text { HIV testing } \\
-\quad \text { Prescribe medicine } \\
\end{array}$ \\
\hline VHTs & \begin{tabular}{|l|} 
Non-technical tasks \\
\end{tabular} \\
\hline
\end{tabular}




\begin{tabular}{|l|ll|}
\hline & - & Record information at health facilities \\
& - & Give health education talks \\
& - & Advise and Counsel women \\
& - & Sensitize and mobilize community \\
& - & Distribute medical resources \\
& - & Follow-up of pregnant women \\
& - & Refer women \\
& Intermediate tasks: \\
\hline Pregna & - Non-technical tasks \\
nt & - & Health talks to fellow women \\
women & - & Birth preparedness \\
& - & Regular checkups \\
\hline
\end{tabular}

\subsection{IT support in task shifting}

IT supports task shifting as an enabler and a trigger in executing tasks as shown in table 3 and elaborated

\section{IT as an enabler}

Reporting and data collection systems are two good examples of how IT plays an enabling role in maternal healthcare. Reporting systems include the district health information system (DHIS2) and the HIV weekly surveillance system (mTrac). DHIS2 is a government-supported system that is used for reporting statistical health data such as admissions, maternal deaths and antenatal care (ANC) visits. The system is used by records officers who collaborate with midwives on data capture. mTrac is an SMS-based system used by midwives and records officers for weekly surveillance of epidemic prone illnesses. In addition, mTrac is used to track maternal deaths and PMTCT indicators that include details of pregnant women who have attended first ANC, tested for HIV, were positive and linked to care. As quoted by the midwife from district A" they trained us and gave us a code $6767 . .$. we send and receive messages from it for free .. it is based on PMTCT indicators to capture positive mothers on mTrac". Weekly data reported in mTrac is used to validate monthly data for DHIS2 thereby improving data quality.

Data collection systems include OpenMRS also referred so as UgandaEMR and Open Data Kit (ODK) which is a mobile app. Open MRS is used to collect data from the Antiretroviral Therapy (ART) clinic, which is concerned with HIV testing and counseling. HIV and TB patients' data is captured including their attendance and reminders for next drug pick up. Aggregated statistics are captured in DHIS2 as reported by a records officer district C "...what OpenMRS does for us, with just a click, it aggregates for you, ... and you put in DHIS2." In addition, ODK is used in maternal healthcare to capture data on family planning in terms of age of recipients and the contraceptives provided to them, based on facility or outreach activities conducted in the community. As quoted by the records officer in District $\mathrm{C}$ "instead of waiting for monthly reports from DHIS2, they want to know on a daily basis...how many are you giving family planning... how many are new acceptors because they need to know those who have just started using modern family planning." It is used for improvement in service provision by finding out how the community responds to different family planning interventions. Weekly reports enable resource planning in that, midwives at health facilities receive drugs before they requisition through a push method. As quoted from the records officer in district $\mathrm{C}$ "... instead of waiting for a month they see ... facility X last week gave us this number according to this $O D K$ report...so it means we need to get contraceptive from facility $Y$ which is not using much of it, we take it to facility $X$. That is where the push method was introduced from."

VHTs and midwives express that IT can improve their work in the task shifting strategy by supporting the storage of family planning and sanitation data.

\section{IT as an enabler and a trigger}

For district A and B, VHTs and midwives use mHealth apps to provide services to pregnant women in the communities. mHealth apps that play a dual role of IT include GetIN and Living goods apps.

GetIN app enables VHTs and midwives to collect data by registering teenage girls in communities and mapping them to health facilities for ANC as quoted by a VHT "we have GetIN apps on phones, they gave it to me and I am actually using it to map teenage girls from 10 to 25 years from villages ...”. The GetIN app also has a triggering role with its functionality to track missed appointments by signaling midwives about girls who miss appointments in order to follow up with them. Pregnant women have not been trained and they do not know how to use the mHealth apps but if trained, it can help to improve service delivery as quoted by a VHT "if pregnant women are trained on how to use GetIN app, it saves our time and resources on transport."

In addition, living goods app plays an enabling role by allowing VHTs to register pregnant women in communities. In addition, the app plays a triggering role by assessing pregnant women and recommending referrals to nearby health facilities.

\section{IT as a trigger}

IT triggers service exchange through diagnosis and communication. IT as a trigger is shown in the case of how VHTs use a mobile based diagnostic app attached to a device (Wekebere app) that assesses the pregnancy condition, senses condition of the baby and notifies the midwife about pregnancy condition, thus triggering service exchange. The app was meant to be used by 
pregnant women as well but they do not have funds to purchase the device, although most of the pregnant women possess mobile phones, as quoted by the midwife from district B " 8 out of 10 pregnant women we work on have phones but they just use them for calling...some do not have funds to buy the device".

IT also triggers service exchange as communications happen among health workers through WhatsApp, SMS and toll-free lines. WhatsApp messages are exchanged among district health management team on urgent health issues that need addressing. SMS messages are used to share information among registered health workers and records officers. As quoted by the records officer in district B "for the code of 6767, the Ministry of health uses it to tell us that there is Cholera in this place, be assertive." Toll free lines have been provided by an implementing partner in district $\mathrm{C}$ to ensure that women have safe deliveries in hospitals. Women and other people call for any maternal healthcare support and they are responded to by midwives and other administrators. As quoted from a records officer at district C "I usually see people calling midwives, for assistance like, somebody is delivering in sugarcane sambas you come and do this, and they rush there."

\section{Table 3. IT systems used to provide services}

\begin{tabular}{|c|c|c|}
\hline Purpose & System name & $\begin{array}{l}\text { Currently used } \\
\text { by }\end{array}$ \\
\hline \multicolumn{3}{|l|}{ IT as an Enabler } \\
\hline $\begin{array}{l}\text { Report statistical } \\
\text { maternal data }\end{array}$ & $\begin{array}{l}\text { DHIS2 } \\
\text { (Computer - } \\
\text { based) }\end{array}$ & $\begin{array}{l}\text { Records officers } \\
\text { in collaboration } \\
\text { with midwives }\end{array}$ \\
\hline $\begin{array}{l}\text { Weekly } \\
\text { surveillance } \\
\text { reports on } \\
\text { epidemic illnesses, } \\
\text { pregnant women } \\
\text { infected with } \\
\text { HIV/AIDS and } \\
\text { maternal deaths }\end{array}$ & $\begin{array}{l}\text { mTrac (SMS } \\
\text { based) }\end{array}$ & $\begin{array}{l}\text { Midwives and } \\
\text { records officers }\end{array}$ \\
\hline $\begin{array}{l}\text { Data collection on } \\
\text { HIV/AIDS } \\
\text { patients }\end{array}$ & $\begin{array}{l}\text { OpenMRS } \\
\text { (Computer - } \\
\text { based) }\end{array}$ & Records officers \\
\hline $\begin{array}{l}\text { Data collection on } \\
\text { family planning } \\
\text { services }\end{array}$ & $\begin{array}{l}\text { Open Data Kit } \\
\text { (ODK) (Mobile } \\
\text { app) }\end{array}$ & Records officers \\
\hline \multicolumn{3}{|c|}{ IT as an Enabler and a Trigger } \\
\hline $\begin{array}{l}\text { Data collection } \\
\text { (through mapping } \\
\text { teenage girls) and } \\
\text { track missed } \\
\text { appointments }\end{array}$ & $\begin{array}{l}\text { GetIN app } \\
\text { (mHealth app) }\end{array}$ & $\begin{array}{l}\text { VHTs and } \\
\text { midwives }\end{array}$ \\
\hline
\end{tabular}

\begin{tabular}{|l|l|l|}
\hline $\begin{array}{l}\text { Data collection } \\
\text { and recommender } \\
\text { for referral }\end{array}$ & $\begin{array}{l}\text { Living goods } \\
\text { app (mHealth } \\
\text { app) }\end{array}$ & VHTs \\
\hline IT as a Trigger & \multicolumn{2}{|l|}{} \\
\hline $\begin{array}{l}\text { Diagnose and } \\
\text { monitor pregnant } \\
\text { woman and fetus }\end{array}$ & $\begin{array}{l}\text { Wekebere App } \\
\text { (mHealth app } \\
\text { and device) }\end{array}$ & VHTs \\
\hline $\begin{array}{l}\text { Communicate } \\
\text { among health } \\
\text { workers and } \\
\text { pregnant women }\end{array}$ & $\begin{array}{l}\text { Communication } \\
\text { tools }\end{array}$ & $\begin{array}{l}\text { Health workers, } \\
\text { records officers, } \\
\text { pregnant women }\end{array}$ \\
\hline
\end{tabular}

\section{Discussion}

The study proposes a model (see Figure 1) that depicts opportunities for IT in the task shifting strategy. The model identifies tasks shifted not only among professionals (senior midwives and midwives), but also from professionals to non-professionals (VHTs and Pregnant women) and among nonprofessionals. Based on the tasks, opportunities for IT as an enabler and as a trigger in executing the tasks are identified as explained below.

\subsection{Non-technical tasks and IT opportunities}

Non-technical tasks that include administrative, data collection, promotional activities, distribution and monitoring are shifted among professionals and nonprofessionals as shown in Figure 1. Results show that IT has played an enabling and triggering role in execution of non-technical tasks but is limited.

IT enables midwives to carry out administrative tasks but is limited on only reporting and data collection. Other administrative tasks such as drug ordering and scheduling have not fully exploited use of IT specifically as a trigger. Results indicate the use of an app that enables weekly reporting and in turn triggers drug replenishment through a push method. This finding has also been reported by Agarwal et al [29] where IT triggers, in form of stock-out signals are used to distribute drugs between facilities and prevent stock outs. We argue that when shifting distribution tasks to non-professionals, IT can trigger service exchange by improving visibility at the health facilities and communities through signaling.

For administrative and promotional activities, results show that IT has triggered service exchange through phone calls, SMS-based messages and WhatsApp. However, collaboration tools that trigger service exchange in form of scheduling tasks and advocating for promotional activities beyond formal health settings have not been exploited, a similar finding noted by Kampmeijer et al [39] on limited use 
of IT in health promotion and primary prevention outside formal programs. Results show that, VHTs and mentor mothers lack health education charts to help them better explain and illustrate health aspects thereby relying on their memories to recall knowledge from trainings. Kampmeijer et al [39] recommend IT an enabler for health promotion, thereby supporting the use of visuals on apps, websites, video consults and webinars. Mobile-phone based videos and direct observation of treatment can be used to disseminate knowledge about diseases [29] hence improving service provision. Other opportunities for IT as a trigger for promotional activities include digitalized guidelines that trigger service exchange during counselling sessions between VHTs and pregnant women, e-health kiosks [25] and online health communities [26] that advocate for health issues, social media tools that trigger community sensitization and mobilization, awareness raising message alerts in form of health tips. However, such IT opportunities have not been explored to support VHTs.

Based on the analysis, such tools can support pregnant women and mentor mothers to execute tasks such as health education talks, birth preparedness and regular ANC checkups, which are supported by community laws and dialogues. Previous research indicates the use of IT to trigger women participation through online communities and e-health kiosks thereby enabling rural patients to share knowledge with peers, manage diseases and build social networks that promote wellness behaviors and preventive care [25], [26]. We argue that mentor mothers can use mHealth apps to monitor women that miss ANC, a task currently done by VHTs, and to promote exercising and dieting activities. IT can trigger participation through improving pregnant women's knowledge and care seeking behaviors [12].

Lastly, results indicate that apps enable and trigger follow-ups and referrals. They also trigger service exchange by diagnosing pregnant women and referring them to facilities. However, these apps are currently being used by VHTs and midwives and not by pregnant women. We argue that research on how these apps can be tailored for use by pregnant women taking into considerations accessibility, usability and affordability of such apps is needed. Agarwal et al [29] indicate the use of mobile-based patient assessment tools that incorporate treatment guidelines for specific health problems as a means of providing patient-side decision support. We argue that such tools can be used by pregnant women and VHTs not only for decision support but to improve promptness and completeness of data collected at the health facilities [29].

\subsection{Intermediate tasks and opportunities for IT}

Intermediate tasks that include supervision and immunization are shifted to professionals (midwives) and non-professionals (VHTs) respectively but results indicate that IT has not been utilized in the execution of these tasks. Based on the analysis, we argue that IT can play a dual role by capturing supervision outcomes and triggering action plans through monitoring and feedback mechanisms. This finding agrees with Deller et al [8], who indicate the need for supervision tools such as performance assessment, remedial education, mentoring and motivation. The use of such tools helps to avoid isolation and frustration among shifted-to cadres such as fellow midwives and CHWs [8]. In addition, IT can enable VHTs to capture immunization records for pregnant women at the health facilities and at the community level as well as, trigger women to go for the next vaccine through SMS alerts. Use of digital immunization registry and SMS reminders have been recommended by researchers [40], [41] as tools that improve immunization coverage and timeliness.

\subsection{Technical tasks and IT opportunities}

Technical tasks are shifted among professionals and include diagnosis, treatment and reviews of women with complicated cases like breach deliveries. Based on the findings, midwives rely on knowledge acquired through trainings and mentorship to carry out the tasks. In addition, guidelines exist at health facilities to support midwives to diagnose and treat women however, they are not continuously updated. IT has not enabled or triggered execution of technical tasks. This finding agrees with previous studies that indicate limited use of triggers, protocols and checklists for timely diagnosis and treatment, yet such tools facilitate patient-centered care [42]. Simple digitalized job aids can trigger service exchange for midwives. Deller et al [8] notes that, to practice new competences, health workers require up-to-date simple job aids and service protocols that are tested for comprehensibility and ease of use. Simple computerized prediction models can also support them to identify and handle complications to ease their work [31].

\subsection{Clinical tasks and IT opportunities}

Clinical tasks include testing and prescribing medicine for pregnant women. Results show that for HIV testing, IT systems have played an enabling role in collecting data on HIV testing for pregnant women during ANC visits to achieve PMTCT. The system is currently used by records officers however, the system can be improved as a trigger to track treatment and drug replenishment through alerting midwives and pregnant women. In addition, although prescription 
requires continuous knowledge acquisition due to medications changes over time, findings show that midwives rely on their knowledge to prescribe for women. IT has not been utilized yet it can reduce cognitive distance [16] for midwives by triggering them to access up-to-date knowledge on prescriptions. For instance, capturing prescriptions and monitoring drugs that worked for specific diseases triggers midwives to acquire more knowledge on prescription.

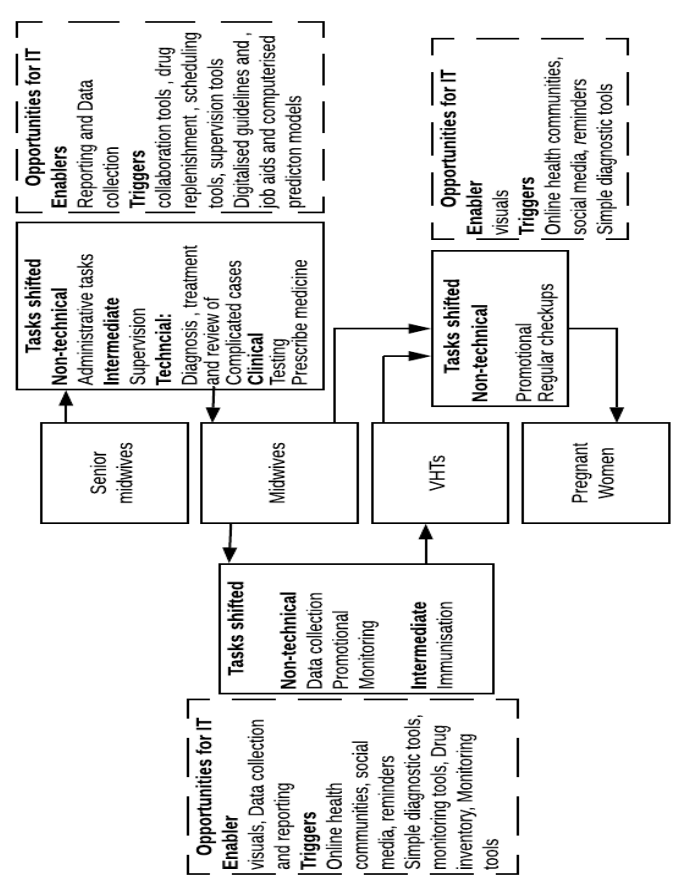

Figure 1. Model depicting opportunities for IT use after tasks are shifted

\section{Conclusion}

The study makes a contribution to research in healthcare transformation, by making visible the interplay of task shifting strategy, through engaging teams of workers in delivering care [10], and the role of IT in maternal healthcare. Particularly, the study contributes to research on the opportunities for IT as a trigger and an enabler for the task shifting strategy to better support healthcare transformation. The study establishes a foundation upon which managerial implications can be suggested for health workers, as a result of IT use in the categorized tasks shifted in maternal healthcare not only to professionals but to non-professionals. The results suggest a more nuanced understanding of the consequences of task shifting beyond the vertical/horizontal dimension and the need to understand the contextual aspects in the professional and non-professional relationships.
The study limitation was that pregnant women were not included in the study, which limited the identification of IT opportunities that would trigger service exchange for pregnant women beyond health workers to include families and friends. We therefore recommend further studies to address this limitation.

\section{References}

[1] WHO, "WHO | Maternal mortality," 2016. [Online]. http://www.who.int/mediacentre/factsheets/fs348/en /. [Accessed: 20-May-2017].

[2] Europe WHO, "Transforming health services delivery towards people-centred health systems," $p$. 23, 2014.

[3] F. Adrodegari and N. Saccani, "Business models for the service transformation of industrial firms," Serv. Ind. J., vol. 37, no. 1, pp. 57-83, 2017.

[4] J. R. McColl-Kennedy et al.,"Health Care Customer Value Cocreation Practice Styles," J. Serv. Res., vol. 15, no. 4, pp. 370-389, 2012.

[5] P. Groves, B. Kayyali, D. Knott, and S. Van Kuiken, "The 'big data'revolution in healthcare:

Accelerating value and innovation," 2013.

[6] J. Das et al., "Rethinking assumptions about delivery of healthcare: Implications for universal health coverage," BMJ, vol. 361, 2018.

[7] WHO, "Everybody's business: strengthening health systems to improve health outcomes: WHO's framework for action.," Production, pp. 1-56, 2007.

[8] B. Deller et al., "Task shifting in maternal and newborn health care: Key components from policy to implementation," Int. J. Gynecol. Obstet., vol. 130, pp. S25-S31, 2015.

[9] R. Braun, C. Catalani, J. Wimbush, and D. Israelski, "Community Health Workers and Mobile Technology: A Systematic Review of the Literature," PLoS One, vol. 8, no. 6, pp. 4-9, 2013.

[10] A. J. Dawson et al., "Task shifting and sharing in maternal and reproductive health in low-income countries: A narrative synthesis of current evidence," Health Policy Plan., vol. 29, no. 3, pp. 396-408, 2014.

[11] A. Aithal and P. S. Aithal, "Task Shifting - An alternative survival strategy for health care organizations," vol. 2, no. 2, pp. 34-48, 2017.

[12] H. Nabudere, D. Asiimwe, and R. Mijumbi, "Task shifting in maternal and child health care: An evidence brief for Uganda," Int. J. Technol. Assess. Health Care, vol. 27, no. 2, pp. 173-179, Apr. 2011.

[13] G. Seidman and R. Atun, "Does task shifting yield cost savings and improve efficiency for health systems? A systematic review of evidence from low-income and middle-income countries," Hum. Resour. Health, vol. 15, no. 1, pp. 1-13, 2017.

[14] W. Hardyman, K. L. Daunt, and M. Kitchener, "Value Co-Creation through Patient Engagement in Health Care: A micro-level approach and research agenda," Public Manag. Rev., vol. 17, no. 1, pp. 90 107, 2015. 
[15] D. H. Lee, "Effects of key value co-creation elements in the healthcare system: focusing on technology applications," Serv. Bus., vol. 13, no. 2, pp. 389-417, 2019.

[16] R. F. Lusch and S. Nambisan, "Service innovation: A service-dominant logic perspective.," Mis $Q$., vol. 39, no. 1, pp. 155-175, 2015.

[17] N. R. Sahni, R. S. Huckman, A. Chigurupati, and D. M. Cutler, "The IT Transformation Health Care Needs," no. December, 2017.

[18] S. S. Harpreet and K. Mcneil, "How is health information technology changing the way we deliver NHS hospital care?," Futur. Healthc. J., vol. 4, no. 2, pp. 117-20, 2017.

[19] A. Frey, M. Trenz, and D. Veit, "The Role of Technology for Service Innovation in Sharing Economy Organizations - a Service- Dominant Logic Perspective" pp. 1885-1901, 2017.

[20] M. A. Akaka and S. L. Vargo, "Technology as an operant resource in service (eco)systems," Inf. Syst. E-bus. Manag., vol. 12, no. 3, pp. 367-384, 2014.

[21] J. R. McColl-Kennedy et al., "The changing role of the health care customer: review, synthesis and research agenda," J. Serv. Manag., vol. 28, no. 1, pp. 2-33, 2017.

[22] J. C. Sweeney, T. S. Danaher, and J. R. McCollKennedy, "Customer Effort in Value Cocreation Activities: Improving Quality of Life and Behavioral Intentions of Health Care Customers," $J$. Serv. Res., vol. 18, no. 3, pp. 318-335, 2015.

[23] H. Nyende, "The role of technology in value cocreation of maternal health care: A service dominant logic perspective," Research Papers. p. 16, 2018.

[24] P. Esmaeilzadeh and M. Sambasivan, "Health Information Exchange (HIE): A literature review, assimilation pattern and a proposed classification for a new policy approach," J. Biomed. Inform., vol. 64, pp. 74-86, 2016.

[25] J. M. Goh, G. Gao, and R. Agarwal, "The Creation of Social Value : Can an Online Health Community Reduce Rural - Urban Health Disparities ?," MIS Q., vol. 40, no. 1, pp. 247-263, 2016.

[26] V. Venkatesh, A. Rai, T. A. Sykes, and R. Aljafari, "Combating Infant Mortality in Rural India: Evidence from a field study of eHealth Kiosk Implementations," MIS Q., vol. 40, no. 2, pp. 353380, 2016.

[27] K. Osei-Frimpong, A. Wilson, and F. Lemke, "Patient co-creation activities in healthcare service delivery at the micro level: The influence of online access to healthcare information," Technol.

Forecast. Soc. Change, 2016.

[28] L. Kooij, W. G. Groen, and W. H. van Harten, "The Effectiveness of Information Technology-Supported Shared Care for Patients With Chronic Disease: A Systematic Review.," J. Med. Internet Res., vol. 19, no. 6, p. e221, Jun. 2017.

[29] S. Agarwal, H. B. Perry, L. A. Long, and A. B. Labrique, "Evidence on feasibility and effective use of mHealth strategies by frontline health workers in developing countries: Systematic review," Tropical Medicine and International Health, vol. 20, no. 8. pp. 1003-1014, 2015.

[30] S. F. V. Sondaal et al., "Assessing the Effect of mHealth Interventions in Improving Maternal and Neonatal Care in Low- and Middle-Income Countries: A Systematic Review," PLoS One, vol. 11, no. 5, p. e0154664, 2016.

[31] B. A. Payne et al., "A Risk Prediction Model for the Assessment and Triage of Women with Hypertensive Disorders of Pregnancy in LowResourced Settings: The miniPIERS (Pre-eclampsia Integrated Estimate of RiSk) Multi-country Prospective Cohort Study," PLoS Med., vol. 11, no. 1, p. e1001589, Jan. 2014.

[32] WHO, PEPFAR, and UNAID, "Task Shifting Global Recommendations and Guidelines," 2008. http://www.who.int/healthsystems/TTRTaskShifting.pdf. [Accessed: 20-May-2017].

[33] C. J. Colvin et al., "A systematic review of qualitative evidence on barriers and facilitators to the implementation of task-shifting in midwifery services," Midwifery, vol. 29, no. 10, pp. 12111221, 2013.

[34] M. S. Dambisya YM, "Policy and Programmatic implication of task shifting in Uganda: A case study. BMC Health Services Research.," BMC Health Serv. Res., vol. 12, no. 61, 2012.

[35] WHO, "Optimizing health worker roles to improve access to key maternal and newborn health interventions through task shifting," World Heal. Organ., pp. 1-98, 2012.

[36] G. Walsham, "Interpretive case studies in IS research: Nature and method," Eur. J. Inf. Syst., vol. 4, no. 2, pp. 74-81, 1995.

[37] WHO, "WHO | Maternal mortality country profiles," 2015.

http://www.who.int/gho/maternal_health/countries /en/. [Accessed: 25-Nov-2017].

[38] V. Braun and V. Clarke, "Using thematic analysis in psychology," Qual. Res. Psychol., vol. 3, no. 2, pp. 77-101, 2006.

[39] R. Kampmeijer, et al., "The use of e-health and mhealth tools in health promotion and primary prevention among older adults: a systematic literature review," BMC Health Serv. Res., vol. 16, no. S5, p. 290, Aug. 2016.

[40] N. T. Nguyen et al., "Digital immunization registry: evidence for the impact of mHealth on enhancing the immunization system and improving immunization coverage for children under one year old in Vietnam.," mHealth, vol. 3, p. 26, 2017.

[41] D. S. Linde et al., "One-way SMS and healthcare outcomes in Africa: Systematic review of randomised trials with meta-analysis," PLoS One, vol. 14, no. 6, pp. e0217485-e0217485, Jun. 2019.

[42] K. S. Arora et al., "Triggers, bundles, protocols, and checklists-what every maternal care provider needs to know," Am. J. Obstet. Gynecol., vol. 214, no. 4, pp. 444-451, Apr. 2016. 